\title{
Obstructive Sleep Apnoea in Children and Adolescents with Ehlers-Danlos Syndrome
}

\author{
Stöberl, Anna S ; Gaisl, Thomas ; Giunta, Cecilia ; Sievi, Noriane A ; Singer, Florian ; Möller,
} Alexander ; Rohrbach, Marianne ; Kohler, Malcolm

\begin{abstract}
BACKGROUND In Ehlers-Danlos syndrome (EDS), a group of monogenic disorders affecting connective tissues, obstructive sleep apnoea (OSA) is highly prevalent in adults. The prevalence of OSA in children with EDS is unknown. OBJECTIVES This prospective cross-sectional study aimed at determining the prevalence of OSA in paediatric EDS patients. METHODS Children with EDS $(\mathrm{n}=24)$ were recruited from the Children's Hospital Zurich and matched to healthy controls. Participants completed home respiratory polygraphy and questionnaires (Sleep-Related Breathing Disorder Scale [SRBD], Epworth Sleepiness Scale [ESS], and Child Health Questionnaire [CHQ]). The American Academy of Sleep Medicine criteria were applied for OSA diagnosis (obstructive apnoea-hypopnoea index [oAHI] 1/h). Conditional logistic regression was used to compare the prevalence of OSA and to adjust for possible confounding. RESULTS OSA was found in $42 \%$ of paediatric EDS patients and in $13 \%$ of matched controls $(\mathrm{OR}=4.5,95 \% \mathrm{CI}=0.97-20.83, \mathrm{p}=0.054)$. The median oAHI was higher in EDS patients than in controls $(0.77 / \mathrm{h}, \mathrm{IQR}=0.19-1.76$, vs. $0.24 / \mathrm{h}, \mathrm{IQR}=0.0-0.60, \mathrm{p}<0.001$ adjusted for age, sex, and BMI z-score). EDS patients had lower scores in most CHQ scales and higher SRBD and ESS scores than controls $(0.26, \mathrm{IQR}=0.1-0.35$, vs. $0.07, \mathrm{IQR}=0-0.19, \mathrm{p}=0.004) ; 7 \pm 4$ vs. $5 \pm 4, \mathrm{p}=0.033$, respectively). CONCLUSION OSA is a previously underestimated EDS-related complication increasing disease burden.
\end{abstract}

DOI: https://doi.org/10.1159/000494328

Posted at the Zurich Open Repository and Archive, University of Zurich

ZORA URL: https://doi.org/10.5167/uzh-160452

Journal Article

Published Version

Originally published at:

Stöberl, Anna S; Gaisl, Thomas; Giunta, Cecilia; Sievi, Noriane A; Singer, Florian; Möller, Alexander; Rohrbach, Marianne; Kohler, Malcolm (2019). Obstructive Sleep Apnoea in Children and Adolescents with Ehlers-Danlos Syndrome. Respiration, 97(4):284-291.

DOI: https://doi.org/10.1159/000494328 


\title{
Clinical Investigations
}

\section{Obstructive Sleep Apnoea in Children and Adolescents with Ehlers-Danlos Syndrome}

\author{
Anna S. Stöberl ${ }^{\mathrm{a}}$ Thomas Gaisl ${ }^{\mathrm{a}} \quad$ Cecilia Giunta ${ }^{\mathrm{c}}$ Noriane A. Sievi ${ }^{\mathrm{a}}$ \\ Florian Singer $^{\mathrm{b}}$ Alexander Möller ${ }^{\mathrm{b}, \mathrm{d}}$ Marianne Rohrbach ${ }^{c}$ Malcolm Kohler ${ }^{\mathrm{a}, \mathrm{d}}$ \\ ${ }^{a}$ Department of Pulmonology, University Hospital Zurich, Zurich, Switzerland; b Division of Respiratory Medicine, \\ University Children's Hospital Zurich, Zurich, Switzerland; 'CConnective Tissue Unit, Division of Metabolism and \\ Children's Research Centre, University Children's Hospital, Zurich, Switzerland; dCentre for Interdisciplinary Sleep \\ Research, University of Zurich, Zurich, Switzerland
}

\section{Keywords}

Obstructive sleep apnoea - Sleep disorders · Rare diseases .

Ehlers-Danlos syndrome $\cdot$ Paediatric patients

\begin{abstract}
Background: In Ehlers-Danlos syndrome (EDS), a group of monogenic disorders affecting connective tissues, obstructive sleep apnoea (OSA) is highly prevalent in adults. The prevalence of OSA in children with EDS is unknown. Objectives: This prospective cross-sectional study aimed at determining the prevalence of OSA in paediatric EDS patients. Methods: Children with EDS $(n=24)$ were recruited from the Children's Hospital Zurich and matched to healthy controls. Participants completed home respiratory polygraphy and questionnaires (Sleep-Related Breathing Disorder Scale [SRBD], Epworth Sleepiness Scale [ESS], and Child Health Questionnaire [CHQ]). The American Academy of Sleep Medicine criteria were applied for OSA diagnosis (obstructive apnoea-hypopnoea index $[\mathrm{oAHI}] \geq 1 / \mathrm{h}$ ). Conditional logistic regression was used to compare the prevalence of OSA and to adjust for possible confounding. Results: OSA was found in
\end{abstract}

\section{KARGER}

() 2018 S. Karger AG, Basel

E-Mail karger@karger.com

www.karger.com/res
$42 \%$ of paediatric EDS patients and in $13 \%$ of matched controls ( $\mathrm{OR}=4.5,95 \% \mathrm{Cl}=0.97-20.83, p=0.054)$. The median OAHI was higher in EDS patients than in controls $(0.77 / \mathrm{h}$, $\mathrm{IQR}=0.19-1.76$, vs. $0.24 / \mathrm{h}, \mathrm{IQR}=0.0-0.60, p<0.001$ adjusted for age, sex, and BMI $z$-score). EDS patients had lower scores in most $\mathrm{CHQ}$ scales and higher SRBD and ESS scores than controls $(0.26, \mathrm{IQR}=0.1-0.35$, vs. $0.07, \mathrm{IQR}=0-0.19, p=$ $0.004) ; 7 \pm 4$ vs. $5 \pm 4, p=0.033$, respectively). Conclusion: OSA is a previously underestimated EDS-related complication increasing disease burden.

(c) 2018 S. Karger AG, Basel

\section{Introduction}

Ehlers-Danlos syndrome (EDS), a group of monogenic disorders affecting connective tissues, is recognized by its fragility of the soft connective tissue and its impact on skin, ligament, joints, blood vessels, and internal organs. With a prevalence of $1 / 5,000$ in the general population, EDS classifies as an orphan disease. However, with increasing medical awareness, underestimation of EDS 
prevalence has been suggested $[1,2]$. Although EDS clinically manifests with symptoms such as hyperelastic skin, joint hypermobility resulting in luxation, joint effusions, abnormal wound healing and scaring, as well as easy bruising, its spectrum also includes unusual and yet mostly unexplained findings such as chronic fatigue and pain, besides neurophysiological and neurodevelopmental features $[1,2]$. Particularly, chronic fatigue occurs as frequently as in $77 \%$ of EDS patients, resulting in a higher level of psychological distress [3]. Investigating this phenomenon more closely, Gaisl et al. [4] found a higher prevalence of obstructive sleep apnoea (OSA) in adults with EDS compared to matched controls, and OSA was associated with fatigue and excessive daytime sleepiness in EDS patients.

In adults, the key symptom of OSA is excessive daytime sleepiness, whereas in children and adolescents, behavioural and cognitive alterations such as hyperactivity and attention-deficit disorder are more frequent consequences [5]. Untreated OSA in developing children is associated with poorer executive function, worse memory skills, and lower general intelligence, resulting in poor school performance and reduced quality of life [6-8]. Furthermore, children with severe OSA are at a higher risk of developing cardiovascular disease including systemic hypertension, pulmonary hypertension, and cor pulmonale with heart failure [9-11]. Moreover, it has been observed that children with severe OSA are at risk of growing poorly and gaining less weight than healthy peers [12].

The estimated prevalence of paediatric OSA in the general population ranges from 2 to $10 \%$, depending on the criteria of diagnosis [13-15]. The prevalence of OSA in children and adolescents with EDS, however, has not yet been assessed, despite multiple risk factors for OSA in these patients. Orofacial anatomic changes and muscular hypotonia as well as abnormal growth of the nasomaxillary complex often seen in EDS patients may lead to increased nasal resistance and altered maxillary development $[1,16]$. In addition, hyperplasia of the adenotonsillar tissue during preschool years may contribute to upper airway obstruction in these children $[1,9,16]$. Therefore, we aimed to assess the prevalence of OSA and its possible association with symptoms and quality of life in children and adolescents with EDS. We hypothesized that OSA is more prevalent in EDS compared to healthy controls. The primary outcome was OSA diagnosed based on current recommendations [17]; the secondary outcomes were symptoms related to sleep-disordered breathing.

\section{Methods}

\section{Subjects}

This study was conducted as a prospective one-to-one-matched case-control study comparing children and adolescents with EDS with healthy controls. From April 2015 to December 2016, children and adolescents with EDS $(n=33)$ aged from 6 to 18 years were recruited from the University Children's Hospital Zurich during ambulatory visits in the Connective Tissue Unit of the Division of Metabolism and Children's Research Centre and via an international network based on EDS associations and EDS support groups. Children and adolescents with EDS were eligible for enrolment if they had a confirmed diagnosis of EDS according to the Villefranche diagnostic criteria [1] or, if applicable, objectively confirmed by gene analysis, pathological electron microscopic results, abnormal biochemical collagen findings, or altered urinary pyridinoline crosslink formation as previously reported by Gaisl et al. [4]. As the study was carried out between April 2015 and December 2016, EDS patients were not diagnosed according to the newest guidelines published in 2017, and nomenclature in this study was used accordingly [18]. EDS patients were matched to healthy controls one-to-one according to sex, age ( \pm 1.5 years), ethnicity, and nutritional status based on BMI $z$-score (severe thinness, thinness, normal weight, overweight, and obesity [19]). Control subjects were recruited via online advertisements and leaflets without mentioning "sleep apnoea" or "sleep disturbances" to minimize possible selection bias. Exclusion criteria were continuous positive airway pressure therapy, pregnancy, as well as physical and intellectual incapacity to adhere to the study protocol. Moreover, sleep studies were not conducted when children showed symptoms suggesting upper airway infections, allergic rhinitis, or topical nasal treatment.

\section{Measurements}

The main outcome of interest was the assessment of the OSA prevalence in children and adolescents with EDS. Other outcomes of interest were predictors of OSA and its impact on quality of life in children and adolescents with EDS.

After enrolment, parents completed a detailed questionnaire together with their child, including socioeconomic characteristics, ethnicity, relevant medical history regarding EDS, history of adenoidectomy or tonsillectomy, respiratory symptoms (rhinitis, cough), asthma, snoring status, cardiac disease, level of activity, and history of smoking and alcohol consumption. Additionally, data concerning participants' parents' history of adenoidectomy, OSA, and smoking habits (e.g., during pregnancy) were collected. The BMI $z$-score was computed using the QuesGen online calculator (http://www.quesgen.com/BMIPedsCalc.php). Neck circumference, gauged at the most prominent part of the thyroid, and waist circumference, measured in the middle between the iliac crest and the lowest rib on the level of the navel, were either selfmeasured as instructed or assessed by a physician (A.S.S.) [20]. Optionally, the participants' tonsillar size and the visibility of anatomical structures in the oropharynx was assessed and documented using the Mallampati score and the Brodsky classification, respectively.

All patients included in the study completed a respiratory polygraphy using an out-of-centre level 3 portable sleep test (ApneaLink Air or Nox T3, both ResMed, San Diego, CA, USA). Sleep recordings always took place in the home environment to ensure 
the child's best quality of sleep, and recordings of paediatric EDS patients and their matched controls were always taken with the same type of device. OSA was diagnosed according to the scoring criteria of the American Academy of Sleep Medicine 2012 with an obstructive apnoea-hypopnoea index (oAHI) $\geq 1 /$ h. OSA syndrome (OSAS) was defined as an oAHI $\geq 1 /$ h plus symptoms suggestive of sleep-disordered breathing. Standard paediatric scoring criteria were used for scoring respiratory events [17]. In particular, obstructive apnoea was scored if the peak signal excursion dropped by $\geq 90 \%$ of the baseline for at least two breaths in the presence of respiratory effort, and hypopnoea was scored if the peak signal excursion dropped by $\geq 30 \%$ for at least two breaths with a desaturation of $\geq 3 \%$ [17]. All sleep studies were reviewed manually by a trained investigator (A.S.S.) and checked by a clinical sleep expert (A.M.). The investigators were blinded with regard to EDS status and results of the questionnaires.

Parents completed validated questionnaires for breathing disorders during sleep utilizing the Sleep-Related Breathing Disorder Scale (SRBD), daytime sleepiness utilizing the Epworth Sleepiness Scale (ESS), and quality of life utilizing the Child Health Questionnaire $(\mathrm{CHQ})$ with their children.

\section{Statistical Methods}

All data was analysed with Stata version 14 (StataCorp LP, College Station, TX, USA). Results are shown as mean (standard deviation) or median and quartiles as appropriate. A two-sided $p$ value $<0.05$ was considered statistically significant.

To compare the prevalence of OSA between children and adolescents with EDS and controls, conditional logistic regression was used, adjusting for possibly confounding variables (age, sex, and BMI $z$-score). For comparison of respiratory events, mixed-effects linear regression was applied, adjusting for the confounding variables and accounting for the cluster of each one-to-one-matched EDS-control pair as a random effect. For analysis of the CHQ, mixed-effects linear regression was calculated in pairs, and for analysis of predictors unadjusted linear regression was used.

\section{Results}

\section{Participants}

Of 49 identified paediatric EDS patients, 33 could be included in the study. Nine patients dropped out of the study, thus the data of 24 paediatric EDS patients matched one-to-one to 24 healthy controls entered the final analysis. The EDS diagnosis was confirmed in all paediatric EDS patients as mentioned in the Methods section, and none of the patients was newly diagnosed due to study participation. The baseline characteristics of paediatric EDS patients and healthy controls are shown in Table 1, and additional background information regarding EDS subtypes, criteria of diagnostic confirmation, medical centres for diagnosis, and countries of origin of paediatric EDS patients can be found in online supplementary Tables 1-4 (for all online suppl. material, see www.karger.com/doi/10.1159/000494328).

OSA in Children and Adolescents with Ehlers-Danlos Syndrome

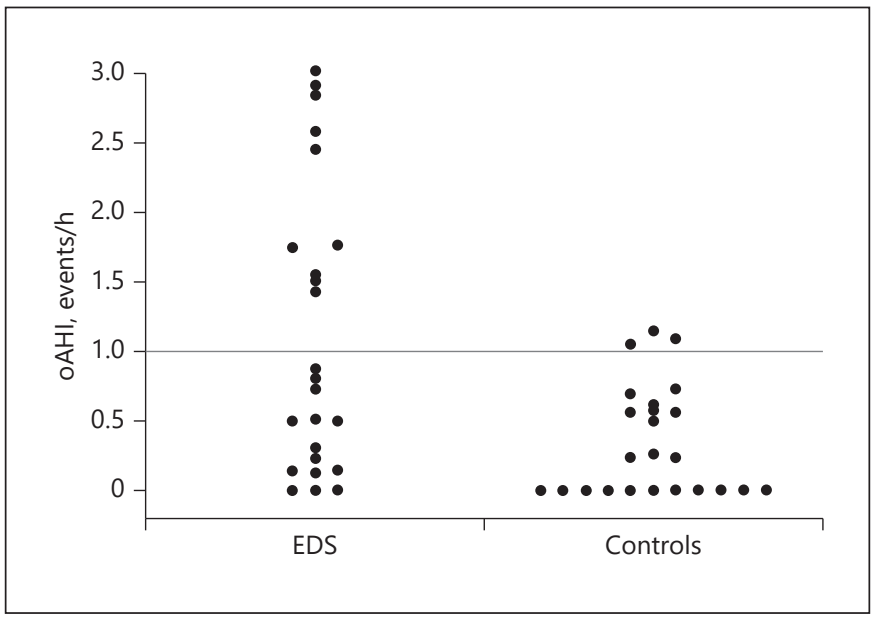

Fig. 1. Distribution of oAHI in paediatric EDS patients and controls. The dashed line indicates the threshold for OSA (oAHI $\geq 1)$. The median (IQR) oAHI was higher in the EDS group (0.77 [0.19$1.76])$ than in the control group $(0.24$ [0.00-0.60]), with $p<0.001$. EDS, Ehlers-Danlos syndrome; oAHI, obstructive apnoea-hypopnoea index; OSA, obstructive sleep apnoea.

Table 1. Baseline characteristics of participants in each group

\begin{tabular}{lcc}
\hline & $\begin{array}{l}\text { EDS } \\
(n=24)\end{array}$ & $\begin{array}{l}\text { Controls } \\
(n=24)\end{array}$ \\
\hline Female sex & $16(66.7 \%)$ & $16(66.7 \%)$ \\
Caucasian ethnicity & $23(95.8 \%)$ & $23(95.8 \%)$ \\
Age, years & $14.2(10.7-15.3)$ & $13.9(10.7-15.9)$ \\
BMI $z$-score & $-0.55 \pm 1.1$ & $-0.24 \pm 1.0$ \\
Neck circumference, cm & $30.7 \pm 3.1$ & $30.9 \pm 2.6$ \\
Waist circumference, cm & $68.1 \pm 7.7$ & $69.9 \pm 8.9$ \\
Neck-to-waist ratio & $0.46 \pm 0.0$ & $0.44 \pm 0.0$ \\
\hline
\end{tabular}

Values are presented as $n(\%)$, median (IQR), or mean \pm SD. EDS patients and controls were matched one-to-one for sex, age, category of BMI $z$-score, ethnicity, and sleep monitoring device. BMI, body mass index; EDS, Ehlers-Danlos syndrome.

\section{Prevalence of OSA}

OSA was found to be highly prevalent in paediatric EDS patients with 42 vs. $13 \%$ in controls with an OR of 4.5 (95\% CI $=0.97-20.83, p=0.054)$. Moreover, in the adjusted analysis for the confounding variables (age, sex, and BMI $z$-score), a significantly higher prevalence of OSA as well as OSAS was seen in paediatric EDS patients. Furthermore, the sleep study data showed a statistically significantly higher median oAHI in paediatric EDS patients than in controls (Table 2; Fig. 1). Obstructive apnoeas and hypopnoeas were more often found in the EDS 
Table 2. Sleep study data for paediatric EDS patients and controls

\begin{tabular}{|c|c|c|c|c|}
\hline OSA $(\mathrm{oAHI} \geq 1)$ & $10(41.7 \%)$ & $3(12.5 \%)$ & 0.011 & $0.07-0.56$ \\
\hline OSAS & $4(16.7 \%)$ & $0(0 \%)$ & 0.025 & $0.02-0.33$ \\
\hline oAHI, h & $0.77(0.19-1.76)$ & $0.24(0.00-0.60)$ & $<0.001$ & $0.40-1.28$ \\
\hline Obstructive apnoeas, events/h & $0.30(0.13-1.00)$ & $0.00(0.00-0.26)$ & 0.002 & $0.18-0.81$ \\
\hline Central apnoeas, events/h & $0.59(0.20-1.15)$ & $0.31(0.00-0.69)$ & 0.119 & $-0.08-0.69$ \\
\hline $\mathrm{SpO}_{2}, \%$ & $95 \pm 1$ & $95 \pm 1$ & 0.914 & $-0.74-0.83$ \\
\hline Minimal SpO $2, \%$ & $90(83-92)$ & $90(86-93)$ & 0.369 & $-6.07-2.26$ \\
\hline Snoring events/h & $5.62(3.63-17.51)$ & $6.20(3.72-10.23)$ & 0.137 & $-1.48-10.79$ \\
\hline Nightly heart rate, bpm & $68 \pm 9$ & $68 \pm 7$ & 0.716 & $-5.03-3.45$ \\
\hline ESS score & $7 \pm 4^{\mathrm{a}}$ & $5 \pm 4$ & 0.033 & $0.22-5.31$ \\
\hline SRBD score & $0.26(0.1-0.35)^{\mathrm{a}}$ & $0.07(0-0.19)$ & 0.004 & $0.04-0.21$ \\
\hline
\end{tabular}

Values are presented as $n(\%)$, median (IQR), or mean \pm SD. All analyses were adjusted for age, sex, and BMI $z$-score. EDS, EhlersDanlos syndrome; ESS, Epworth Sleepiness Scale; oAHI, obstructive apnoea-hypopnoea index; OSA, obstructive sleep apnoea; OSAS, obstructive sleep apnoea syndrome; $\mathrm{SpO}_{2}$, oxygen saturation; $\mathrm{SRBD}$, Sleep-Related Breathing Disorder Scale. ${ }^{\mathrm{a}} n=20$.

Fig. 2. Distribution of the frequency of OSA diagnosis by EDS subtypes in total numbers. EDS, Ehlers-Danlos syndrome; OSA, obstructive sleep apnoea.

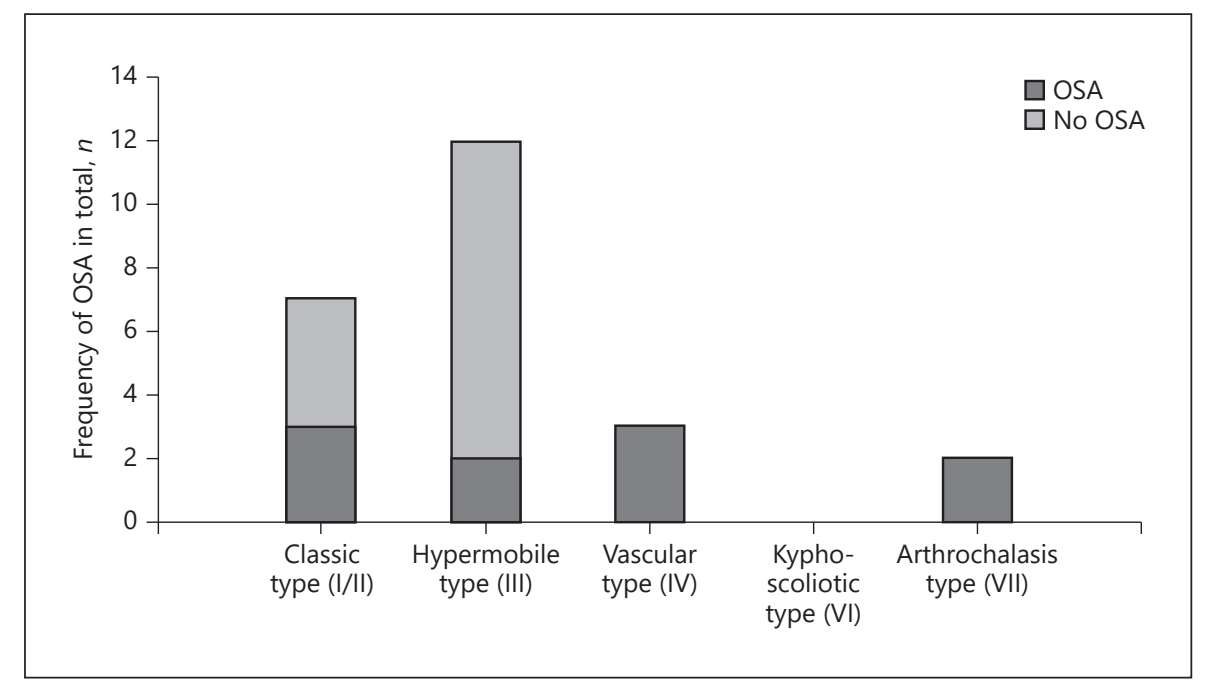

group, whereas central apnoeas occurred similarly often in both groups. Additionally, no differences between groups were found regarding snoring events, mean nightly heart rate, and mean and minimal oxygen saturation. The complete analysed sleep study data can be found in Table 2. Subgroup analysis showed no differences in the frequency of OSA between different types of EDS (95\% $\mathrm{CI}=-0.30-2.44, p=0.119)$ (online suppl. Table 5; Fig. 2).

Daytime sleepiness measured by ESS and sleep-related breathing disorders measured by the SRBD were significantly higher in the EDS group, and an unadjusted analy- sis showed a modest correlation between a higher oAHI and a higher ESS score $(r=0.317,95 \% \mathrm{CI}=0.00-0.11$, $p=0.039$ ).

\section{Quality of Life}

Children and adolescents with EDS had significantly lower scores in most CHQ scales than controls $(p<0.05)$, except for the behaviour categories, mental health, parental impact - time, and family cohesion (Table 3; Fig. 3). Generally, a higher oAHI was associated with a lower general health perception $(r=-0.357,95 \% \mathrm{CI}=-0.02$ to 
Table 3. Detailed CHQ results

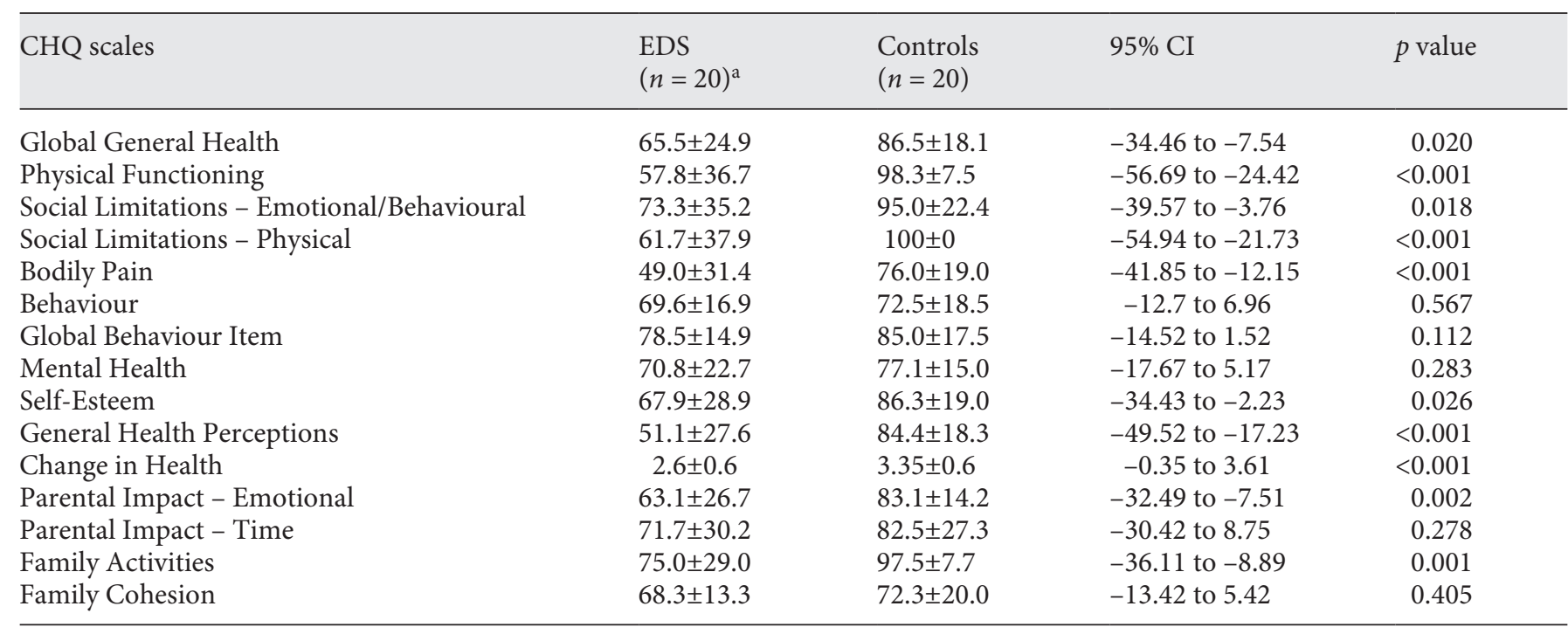

Values are presented as mean \pm SD. CHQ, Child Health Questionnaire; EDS, Ehlers-Danlos syndrome. ${ }^{\text {a }} 20$ EDS patients completed the validated CHQ questionnaire with their parents; analyses were calculated in matched pairs.

$-0.00, p=0.017)$ and lower scores in the categories physical functioning $(r=-0.381,95 \% \mathrm{CI}=-0.02$ to $-0.00, p=$ $0.011)$ and both emotional $(r=-0.314,95 \% \mathrm{CI}=-0.02$ to $-0.00, p=0.038)$ and physical social limitations $(r=$ $-0.411,95 \% \mathrm{CI}=-0.02$ to $-0.00, p=0.006)$. OSA diagnosis correlated with lower physical functioning $(r=-0.334$, $95 \% \mathrm{CI}=-0.01$ to $-0.00, p=0.027)$.

\section{Predictors of OSA}

Potential predictors of a high oAHI in children and adolescents with EDS were investigated (Table 4). BMI $z$-score, neck-to-waist ratio, snoring history, history of tonsillectomy or adenectomy, or asthma did not predict OSA. In the unadjusted model, younger age correlated with a higher oAHI $(p=0.030)$. However, after adjusting the model for other potential predictive factors, age was no longer independently associated with oAHI.

\section{Discussion}

This was the first study to investigate the prevalence of OSA, its predictors, and its impact on quality of life in paediatric EDS patients. We found a substantially higher frequency of OSA and OSAS in children and adolescents with EDS in comparison to healthy matched controls. OSA diagnosis was associated with impaired quality of

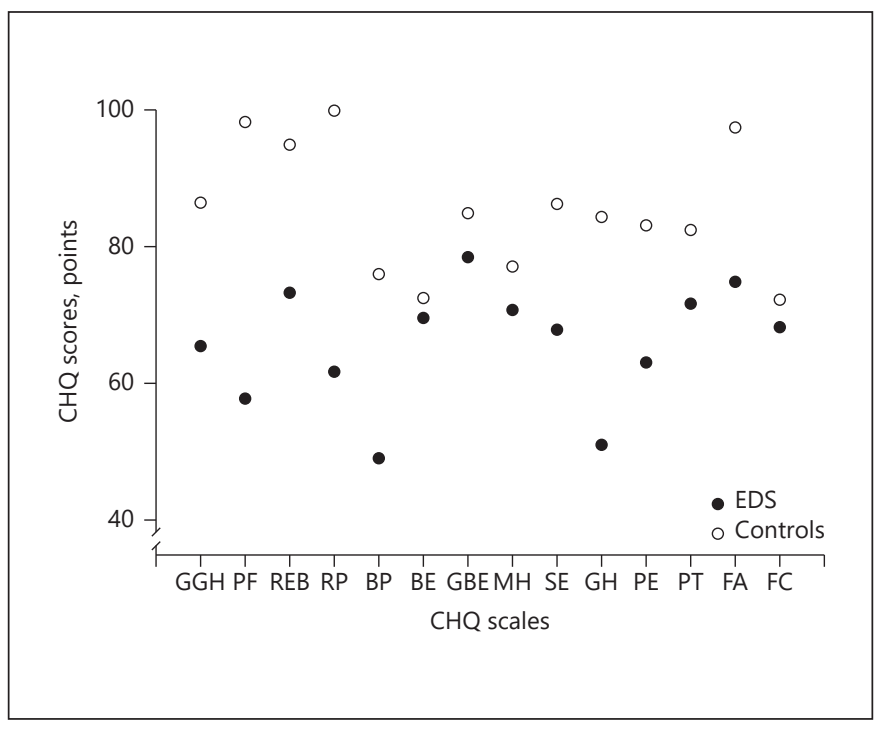

Fig. 3. Differences in the median scores of the CHQ between children and adolescents with EDS and controls. The maximum acquirable score is 100 in each scale, except for Change in Health, which was excluded from the graph due to its categorical values $(2.6 \pm 0.6$ vs. $3.35 \pm 0.6)$. The scales are as follows: GGH, Global General Health; PF, Physical Functioning; REB, Social Limitations - Emotional/Behavioural; RP, Social Limitations - Physical; BP, Bodily Pain; BE, Behaviour; GBE, Global Behaviour Item; $\mathrm{MH}$, Mental Health; SE, Self-Esteem; GH, General Health Perceptions; PE, Parental Impact - Emotional; PT, Parental Impact - Time; FA, Family Activities; FC, Family Cohesion. CHQ, Child Health Questionnaire; EDS, Ehlers-Danlos syndrome. 
Table 4. Unadjusted analysis of predictors of a higher oAHI in EDS patients

\begin{tabular}{|c|c|c|c|c|c|}
\hline & & \multicolumn{2}{|l|}{ Unadjusted } & \multicolumn{2}{|l|}{ Adjusted $^{\mathrm{a}}$} \\
\hline & & $95 \% \mathrm{CI}$ & $p$ value & $95 \% \mathrm{CI}$ & $p$ value \\
\hline \multicolumn{6}{|l|}{$n=24$} \\
\hline Female sex & $16(66 \%)$ & -1.61 to 0.18 & 0.110 & -2.39 to 1.06 & 0.381 \\
\hline Age, years & $14.2(10.7-15.3)$ & -0.26 to -0.01 & 0.030 & -0.28 to 0.29 & 0.977 \\
\hline BMI $z$-score & $-0.55 \pm 1.1$ & -0.12 to 0.68 & 0.159 & -1.15 to 0.85 & 0.725 \\
\hline Neck-to-waist ratio & $0.46 \pm 0.0$ & -29.26 to 4.58 & 0.142 & -31.41 to 18.59 & 0.553 \\
\hline Asthma diagnosis & $5(20.8 \%)$ & -1.68 to 0.45 & 0.246 & -1.39 to 2.18 & 0.608 \\
\hline \multicolumn{6}{|l|}{$n=20$} \\
\hline Snoring history & $9(45 \%)$ & -0.65 to 1.18 & 0.555 & -1.50 to 1.46 & 0.972 \\
\hline History of tonsillectomy/adenectomy & $1(5 \%)$ & -2.93 to 1.20 & 0.390 & -3.29 to 1.93 & 0.549 \\
\hline Exposure to cigarettes at home & $0(0 \%)$ & - & - & & \\
\hline Prenatal cigarette exposure & $0(0 \%)$ & - & - & & \\
\hline OSA diagnosis in parents ${ }^{\mathrm{b}}$ & $3(16.7 \%)$ & -0.05 to 2.28 & 0.060 & -1.45 to 4.04 & 0.294 \\
\hline
\end{tabular}

Values are presented as $n(\%)$, median (IQR), or mean \pm SD. Bold indicates statistical significance $(p<0.05)$. BMI, body mass index; EDS, Ehlers-Danlos syndrome; oAHI, obstructive apnoea-hypopnoea index; OSA, obstructive sleep apnoea. ${ }^{a}$ Adjusted for sex, age, BMI $z$-score, neck-to-waist ratio, diagnosis of asthma, history of tonsillectomy or adenectomy, and OSA diagnosis in parents. $R^{2}=0.6110 .{ }^{\mathrm{b}} n=18$.

life and lower physical functioning. Our study reveals that OSA and OSAS have previously been underestimated as an EDS-related complication that significantly increases disease burden.

In the present study, we observed that $42 \%$ of paediatric EDS patients suffered from OSA and 17\% from OSAS. In comparison to adult EDS patients, the prevalence of OSA in children was considerably higher, but OSAS was less often observed; in 34\% of adults with EDS OSA was diagnosed, and $23 \%$ suffered from OSAS [4]. Compared to adults with EDS, this may be related to poor symptom perception in both children and parents. Moreover, in our study, the prevalence of OSA in children and adolescents with EDS was substantially higher than in matched controls (13\%). Similar to our findings, a study from the US observed an oAHI $\geq 1$ in $10.8 \%$ of children aged $8-11$ years [21]. In comparison to other diseases associated with a high OSA prevalence in children, it is notable that the prevalence of OSA in children and adolescents with EDS was found to be even higher than in children and adolescents with e.g. cleft palate $(8.5 \%$ with an oAHI $\geq 1)$ [22], but lower than in children with Pierre Robin syndrome ( $85 \%$ with a oAHI $\geq 1)$ or Down syndrome $(73 \%$ with an oAHI $\geq 1)[23,24]$. In contrast to children suffering from these pathologies, in EDS patients craniofacial abnormalities are not risk factors for OSA [4]. Genetically related cartilage defects and tissue flaccidity that in- creases the pharyngeal collapsibility, on the other hand, are specific for EDS and suspected to cause OSA [16, 25].

Awareness about the high prevalence of OSA in children and adolescents with EDS is important in the clinical setting. The diagnosis of sleep disorders through sleep studies is costly and often cumbersome, especially for young children. Therefore, the identification of potential predictors would be of great help for assessing possible OSA and the need for a sleep study. In the general population, shifts in the risk factors for OSA occur from childhood through adolescence [21]. While in childhood small airway size and enlarged lymphoid tissue increase the risk, in adolescence obesity and male sex are associated with OSA [26]. However, in our study population, children and adolescents were thin with a mean BMI $z$-score of -0.55 , which is considered a low normal nutritional status, and predominantly female (67\%), which is consistent with proportions reported by other EDS studies [4]. Nevertheless, tonsillar hypertrophy being more frequent in younger children, this might explain why younger children were at higher risk of developing OSA than adolescents in our study. Nonetheless, we could not find a relationship between history of tonsillectomy or adenectomy and a higher oAHI in the EDS group.

In our study population, $50 \%(n=12)$ of EDS patients suffered from the hypermobile EDS subtype (EDS-HT), and 6 patients with this subtype were diagnosed clini- 
cally by the Villefranche criteria. EDS-HT and joint hypermobility syndrome, a disorder with hypermobility, laxity, and instability of the joints, are clinically very similar [27]. EDS patients as well as joint hypermobility syndrome patients suffer frequently from fatigue $[3,28]$. Yet, our study was underpowered for subgroup analysis. Fatigue, on the other hand, is known to contribute to impaired quality of life [28]. In our study, children and adolescents with EDS were found to suffer more often from daytime sleepiness and sleep-related breathing disorders than their control peers. Furthermore, daytime sleepiness was associated with a lower general health perception and social limitations due to physical health problems in the CHQ score. Similarly, in adolescents with joint hypermobility syndrome aged $12-18$ years, correlations between increased general fatigue and emotional and social function impairment were found [29]. In our study, OSA had an impact on children and adolescents in general, as participants diagnosed with OSA presented with lower scores in physical functioning and a higher oAHI correlated with lower general health perception and emotional and physical social limitations. It is remarkable that both increased daytime sleepiness in paediatric EDS patients and diagnosis of OSA in the total study population were associated with lower scores in general health perception. Increased daytime sleepiness and higher oAHI were associated with social limitations due to physical health problems. Further and larger studies are needed to confirm the impact of OSA on overall health in paediatric EDS patients.

Some limitations of our study should be mentioned. We did not use polysomnography for the assessment of childhood OSA, but used respiratory polygraphy. Thereby, we were unable to assess sleep stages, and there is the possibility of an underestimation of the amount of sleepdisordered breathing [30]. However, sleep monitoring at home may be more representative, as children's sleep in a hospital environment was reported to be atypical in one-third of patients [31]. Moreover, the used sleep monitoring devices ApneaLink Air and Nox T3 demonstrated good diagnostic performances in comparison to polysomnography in several studies [32, 33].

\section{Conclusion}

The results of our study demonstrate that there is a high prevalence of OSA(S) in children and adolescents with EDS. Moreover, daytime sleepiness - one of the major symptoms in adult EDS patients - and hyperactivity

were also found to be a problem in children and adolescents with EDS. To assess whether treatment of OSA also improves these symptoms, further studies are needed.

\section{Acknowledgements}

We thank all the children, their parents, and the EDS organizations for their participation and support; without them this study would not have been possible. This work was supported by grants from "Lunge Zürich" and "Lungenliga Schweiz."

\section{Statement of Ethics}

The study was approved by the Cantonal Ethics Committee Zurich, Switzerland (KEK-ZH 2015-0144) and is registered at http://www.ClinicalTrials.gov, NCT02712060. Detailed written information about the study was provided to all legal guardians and an adapted short version was provided to all children and adolescents. Parents or primary caregivers gave written consent on behalf of their children before study participation.

\section{Disclosure Statement}

The authors have no financial relationships and no conflicts of interest relevant to this article to disclose.

\section{Author Contributions}

Conception and design: A.S. Stöberl, T. Gaisl, M. Rohrbach, and M. Kohler. Administrative support: A.S. Stöberl, T. Gaisl, N.A. Sievi, and C. Giunta. Provision of study materials or patients: A.S. Stöberl, T. Gaisl, M. Kohler, C. Giunta, and M. Rohrbach. Collection and assembly of data: A.S. Stöberl, T. Gaisl, and N.A. Sievi. Data analysis and interpretation: A.S. Stöberl, T. Gaisl, F. Singer, and A. Möller. Manuscript writing and final approval of the manuscript: all authors.

References

Respiration

DOI: $10.1159 / 000494328$
1 Steinmann B. The Ehlers-Danlos Syndrom. Connective Tissue and its Heritable Disorders. New York: Wiley-Liss; 2002.

2 Castori M. Ehlers-Danlos syndrome(s) mimicking child abuse: is there an impact on clinical practice? Am J Med Genet C Semin Med Genet. 2015 Dec;169(4):289-92.

3 Voermans NC, Knoop H, van de Kamp N, Hamel BC, Bleijenberg G, van Engelen BG. Fatigue is a frequent and clinically relevant problem in Ehlers-Danlos Syndrome. Semin Arthritis Rheum. 2010 Dec;40(3):267-74.

4 Gaisl T, Giunta C, Bratton DJ, Sutherland K, Schlatzer C, Sievi N, et al. Obstructive sleep apnoea and quality of life in Ehlers-Danlos syndrome: a parallel cohort study. Thorax. 2017 Aug;72(8):729-35. 
5 Melendres MC, Lutz JM, Rubin ED, Marcus CL. Daytime sleepiness and hyperactivity in children with suspected sleep-disordered breathing. Pediatrics. 2004 Sep;114(3):76875.

6 Gottlieb DJ, Chase C, Vezina RM, Heeren TC, Corwin MJ, Auerbach SH, et al. Sleep-disordered breathing symptoms are associated with poorer cognitive function in 5-year-old children. J Pediatr. 2004 Oct;145(4):458-64.

7 Mitchell RB, Kelly J. Behavior, neurocognition and quality-of-life in children with sleepdisordered breathing. Int J Pediatr Otorhinolaryngol. 2006 Mar;70(3):395-406.

8 Hunter SJ, Gozal D, Smith DL, Philby MF, Kaylegian J, Kheirandish-Gozal L. Effect of Sleep-disordered Breathing Severity on Cognitive Performance Measures in a Large Community Cohort of Young School-aged Children. Am J Respir Crit Care Med. 2016 Sep; 194(6):739-47.

9 Marcus CL, Greene MG, Carroll JL. Blood pressure in children with obstructive sleep apnea. Am J Respir Crit Care Med. 1998 Apr; 157(4 Pt 1):1098-103.

10 Chang SJ, Chae KY. Obstructive sleep apnea syndrome in children: Epidemiology, pathophysiology, diagnosis and sequelae. Korean J Pediatr. 2010 Oct;53(10):863-71.

11 Kwok KL, Ng DK, Cheung YF. BP and arterial distensibility in children with primary snoring. Chest. 2003 May;123(5):1561-6.

12 Williams EF 3rd, Woo P, Miller R, Kellman RM. The effects of adenotonsillectomy on growth in young children. Otolaryngol Head Neck Surg. 1991 Apr;104(4):509-16.

13 Sánchez-Armengol A, Fuentes-Pradera MA, Capote-Gil F, García-Díaz E, Cano-Gómez S, Carmona-Bernal C, et al. Sleep-related breathing disorders in adolescents aged 12 to 16 years: clinical and polygraphic findings. Chest. 2001 May;119(5):1393-400.

14 Rosen CL, Larkin EK, Kirchner HL, Emancipator JL, Bivins SF, Surovec SA, et al. Prevalence and risk factors for sleep-disordered breathing in 8- to 11-year-old children: association with race and prematurity. J Pediatr. 2003 Apr;142(4):383-9.
15 Redline S, Tishler PV, Schluchter M, Aylor J, Clark K, Graham G. Risk factors for sleep-disordered breathing in children. Associations with obesity, race, and respiratory problems. Am J Respir Crit Care Med. 1999 May;159 (5 Pt 1):1527-32.

16 Guilleminault C, Primeau M, Chiu HY, Yuen KM, Leger D, Metlaine A. Sleep-disordered breathing in Ehlers-Danlos syndrome: a genetic model of OSA. Chest. 2013 Nov; 144(5): 1503-11.

17 Berry RB, Budhiraja R, Gottlieb DJ, Gozal D, Iber C, Kapur VK, et al.; American Academy of Sleep Medicine; Deliberations of the Sleep Apnea Definitions Task Force of the American Academy of Sleep Medicine. Rules for scoring respiratory events in sleep: update of the 2007 AASM Manual for the Scoring of Sleep and Associated Events. J Clin Sleep Med. 2012 Oct;8(5):597-619.

18 Tinkle B, Castori M, Berglund B, Cohen $\mathrm{H}$, Grahame R, Kazkaz H, et al. Hypermobile Ehlers-Danlos syndrome (a.k.a. Ehlers-Danlos syndrome Type III and Ehlers-Danlos syndrome hypermobility type): clinical description and natural history. Am J Med Genet C Semin Med Genet. 2017 Mar;175(1):48-69.

19 WHO Multicentre Growth Reference Study Group. WHO Child Growth Standards: Length/height-for-age, weight-for-age, weightfor-length, weight-for-height and body mass index-for-age: Methods and development. Geneva: World Health Organization; 2006.

20 Katz SL, Vaccani JP, Barrowman N, Momoli F, Bradbury CL, Murto K. Does neck-to-waist ratio predict obstructive sleep apnea in children? J Clin Sleep Med. 2014 Dec;10(12):1303-8.

21 Spilsbury JC, Storfer-Isser A, Rosen CL, Redline S. Remission and incidence of obstructive sleep apnea from middle childhood to late adolescence. Sleep (Basel). 2015 Jan;38(1):23-9.

22 Robison JG, Otteson TD. Increased prevalence of obstructive sleep apnea in patients with cleft palate. Arch Otolaryngol Head Neck Surg. 2011 Mar;137(3):269-74.

23 Anderson IC, Sedaghat AR, McGinley BM, Redett RJ, Boss EF, Ishman SL. Prevalence and severity of obstructive sleep apnea and snoring in infants with Pierre Robin sequence. Cleft Palate Craniofac J. 2011 Sep; 48(5):614-8.
24 Hill CM, Evans HJ, Elphick H, Farquhar M, Pickering RM, Kingshott R, et al. Prevalence and predictors of obstructive sleep apnoea in young children with Down syndrome. Sleep Med. 2016 Nov-Dec;27-28:99-106.

25 Malfait F, Wenstrup RJ, De Paepe A. Clinical and genetic aspects of Ehlers-Danlos syndrome, classic type. Genet Med. 2010 Oct; 12(10):597-605.

26 Schwengel DA, Dalesio NM, Stierer TL. Pediatric obstructive sleep apnea. Anesthesiol Clin. 2014 Mar;32(1):237-61.

27 Castori M, Tinkle B, Levy H, Grahame R, Malfait F, Hakim A. A framework for the classification of joint hypermobility and related conditions. Am J Med Genet C Semin Med Genet. 2017 Mar;175(1):148-57.

28 Krahe AM, Adams RD, Nicholson LL. Features that exacerbate fatigue severity in joint hypermobility syndrome/Ehlers-Danlos syndrome - hypermobility type. Disabil Rehabil. 2018 Aug;40(17):1989-96.

29 Zekry OA, Ahmed MA, Elwahid HA. The impact of fatigue on health related quality of life in adolescents with benign joint hypermobility syndrome. Egypt Rheumatol. 2013 Apr;35: 77-85.

30 Tan HL, Gozal D, Ramirez HM, Bandla HP, Kheirandish-Gozal L. Overnight polysomnography versus respiratory polygraphy in the diagnosis of pediatric obstructive sleep apnea. Sleep (Basel). 2014 Feb;37(2):255-60.

31 Das S, Mindell J, Millet GC, Ofer D, Beck SE, Mason TB, et al. Pediatric polysomnography: the patient and family perspective. J Clin Sleep Med. 2011 Feb;7(1):81-7.

32 Cairns A, Wickwire E, Schaefer E, Nyanjom D. A pilot validation study for the NOX T3(TM) portable monitor for the detection of OSA. Sleep Breath. 2014 Sep;18(3):609-14.

33 El Shayeb M, Topfer LA, Stafinski T, Pawluk L, Menon D: Diagnostic accuracy of level 3 portable sleep tests versus level 1 polysomnography for sleep-disordered breathing: a systematic review and meta-analysis. CMAJ. 2014 Jan;186(1):E25-51. 